\title{
Die gerichtsmedizinische Wundbegutachtung vom 16. zum 18. Jahrhundert
}

\author{
Von Esther Fischer-Homberger
}

Bis zum 19. Jahrhundert war die zentrale Frage an den Wundbegutachter diejenige nach dem Grad der «Tödlichkeit» gegebener Wunden. Und die Antwort der ärztlichen Experten pflegte zugleich eine Antwort auf die Schuldfrage zu sein: je «tödlicher» eine Verletzung, desto schuldiger deren Verursacher an ihren unglücklichen Folgen.

Die Tödlichkeit von Verletzungen wurde nun zu verschiedenen Zeiten verschieden bestimmt - zunächst vorwiegend prognostisch, später zunehmend pathologisch-anatomisch und schließlich auch unter physiologischen Gesichtspunkten. Diese Entwicklung soll hier kurz nachgezeichnet werden.

Zunächst also versuchten die als Experten befragten Ärzte die Tödlichkeit von Verletzungen prognostisch zu beantworten. Sie stellten sich damit in antike Tradition - Celsus hatte über die Wunden geschrieben, der Arzt müsse "vor allem wissen, welche unheilbar, welche schwer und welche leichter zu heilen sind» ${ }^{1}$-, wobei sie «unheilbar - heilbar» mit «tödlich nichttödlich» gleichsetzten ${ }^{2}$.

So beurteilten jene Bologneser Ärzte des späten 13. und des früheren 14. Jahrhunderts, deren Berichte uns als früheste gerichtsmedizinische Expertisen bekannt sind, die ihnen vorgelegten Fälle, als ob sie sich auf eines jener frühen Wundenmann-Bilder gestützt hätten, auf welchen Wunden verschiedener äußerer Lokalisation einfach in heilbare («curabilis») und unheilbare («incurabilis») unterteilt sind ${ }^{3}$. «Zunächst in der Brust: sieben tödliche Wunden, desgleichen im Unterbauch: eine tödliche Wunde ... im rechten Kinnbacken eine nicht tödliche Wunde ...»: dies beschworen zwei Chirurgen im Februar 1298 zu Bologna ${ }^{4}$. In derselben Tradition schreibt Ambroise Paré als Autor der wohl frühesten gedruckten gerichtsmedizinischen Schrift von allgemeinerer Bedeutung (1575), es müsse der junge Chirurge, welcher vor Gericht über den Tod Verwundeter auszusagen habe, vor allem das Prognostizieren beherrschen ${ }^{5}$. Ebenso sind die Wundbegutachtungslehren eines Bernhardus Suevus (1629 - «ältester Autor über gerichtliche Medicin in Deutschland») ${ }^{6}$ - und eines Melchior Sebitz, den Suevus als seinen Lehrer bezeichnet $(1632-37)^{7}$, durchaus prognostisch orientiert. 
Dieses Verfahren konnte indessen auf die Dauer nicht befriedigen. Denn einmal ist es ein fragwürdiges Unterfangen, am bereits Verstorbenen noch eine Prognose zu stellen - und sehr häufig hatte der Gerichtsarzt es mit Toten zu tun. Zum anderen pflegt die Prognose einer Verletzung durch Faktoren mitbestimmt zu sein, welche dem Verursacher dieser Verletzung nicht zur Last gelegt werden können: für Celsus sind Alter, Konstitution und Lebensweise des Verletzten und äußere Faktoren wie die Jahreszeit von prognostischer Bedeutung, und Paré folgt ihm hierin weitgehend ${ }^{8}$. So behandeln Giovanni Battista Codronchi (1593) ${ }^{9}$ und Fortunatus Fidelis (1602) ${ }^{10}$ die (prognostisch orientierte) Begutachtung Lebender und diejenige Toter getrennt. Codronchi scheint die Frage nach der Prognostik von derjenigen nach der Schuld des Täters zu unterscheiden: Damit er nicht aus Schuldigen Unschuldige und aus Unschuldigen Schuldige mache, schreibt er, müsse der Arzt bei toten Verletzten feststellen, ob der Tod durch eine tödliche $W$ unde oder, bei nichttödlichen Wunden, anders (etwa durch eine Säfteverderbnis) verursacht sei ${ }^{11}$. Fidelis macht die Tödlichkeit einer Wunde ausschließlich von der Natur der verletzten Teile abhängig, ausführlich beschreibt er, welche Strukturen nur mit tödlichen Folgen verletzt werden können. Trotzdem bleibt auch er der Prognostik verhaftet, indem auch er nicht etwa durch Sektion anatomisch bestimmt, welche Organe durch eine Verletzung betroffen seien, sondern symptomatologisch. Als ob er es mit lebenden Verletzten zu tun hätte, beschreibt er, offensichtlich an Celsus orientiert, welche Erscheinungen auf die Verletzung welcher Körperteile hindeuten ${ }^{12}$.

Zum expliziten Bruch mit der prognostischen Tradition kam es in der zweiten Hälfte des 17. Jahrhunderts in Leipzig. 1660 publizierte der dortige Professor der Anatomie und Chirurgie und Stadtphysikus Gottfried Welsch sein «Rationale vulnerum lethalium judicium», wo er ausdrücklich forderte, daß zu begutachtende Wunden zuerst von außen, dann an der geöffneten Leiche untersucht werden, und daß seziert werde, auch wenn man von außen nicht das geringste sehe. 1689 erschien, ebenfalls in Leipzig, des gelehrten Johannes Bohn Schrift über die Untersuchung der tödlichen Wunden. Bohn, ebenfalls Professor der Anatomie in Leipzig und Nachfolger Welschs als dortiger Stadtphysikus, nennt nun die Kunst der Begutachtung tödlicher Wunden ausdrücklich nicht prognostisch, sondern pathologisch-anatomisch («anatomiae practicae seu pathologicae specimen»), und dementsprechend auch sicherer als die Begutachtung von Verletzungen am noch Lebenden ${ }^{13}$. So ist pathologisch-anatomisches Denken definitiv und explizit in die Gerichtsmedizin der Wunden eingeführt und benannt worden, 
Generationen bevor dies für die Gesamtmedizin der Fall gewesen ist («Erst am Ende des 18. Jahrhunderts», schreibt Fritz Hartmann, «verdrängte die Diagnose, das Erkennen der Krankheit, die Prognose ...» ${ }^{14}$, und erst im Todesjahr Morgagnis, 1771, wurde nach Michler «der neuen Forschungsrichtung der Name Pathologische Anatomie gegeben» ${ }^{15}$ ). Daß hier - und nicht nur hier -- eine allgemeine medizinhistorische Entwicklung durch die gerichtliche Medizin vorweggenommen oder eingeleitet wurde, hängt wohl unter anderem damit zusammen, daß der Auftrag, vor Gericht auszusagen, zur Systematisierung von Wissen und zu Theorienbildung anregt und daß in der kontroversen gerichtlichen Situation kritikfester ausgesagt werden muß als in der privatärztlichen.

Doch Bohn ging über die rein pathologisch-anatomische Letalitätsbestimmung bereits hinaus in Richtung physiologischen Denkens. Gerade die systematische Untersuchung von an Verletzungen Verstorbenen mußte ja zeigen, daß tödliche Verletzungen nicht immer auf Läsionen bestimmter innerer Organe oder Strukturen beruhen. Aus der Verletzung scheinbar unwichtiger Gefäße konnten tödliche innere Blutungen erfolgen; stumpfe Schläge, z.B. in den Bauch, konnten offenbar töten, ohne auffällige pathologisch-anatomische Befunde zu hinterlassen. Anstelle der Frage nach dem verletzten Organ mußte daher zunehmend diejenige nach der verletzten Funktion treten. Hatten Hirn-, Herz- und Leberverletzungen ursprünglich als tödlich gegolten, weil sie die «vornehmsten» unter den Organen betrafen ${ }^{16}$, so wurden sie nun tödlich, weil sie diese an der Ausübung ihrer lebenswichtigen Funktionen hinderten. Damit mußte übrigens die alte Unterscheidung zwischen Verletzungen der vornehmsten Organe und denjenigen lebenswichtiger Organe wegfallen ${ }^{17}$. Tatsächlich bezeichnet Bohn als tödlich einfach diejenigen Verletzungen, welche die Bewegungen der Säfte und die Lebensfunktionen so sehr stören, daß das Leben erlischt. Entsprechend mußte eine Wunde für ihn nicht mehr in einer Gewebsdurchtrennung bestehen, wie dies bis dahin selbstverständlich gewesen war ${ }^{18}$. Auch Luxationen, Quetschungen und Erschütterungen, etwa des Hirns oder des Oberbauchs, konnten ja lebenswichtige Funktionen lahmlegen und damit tödlich wirken ${ }^{19}$.

Diese physiologische Orientierung der Wundbegutachtung sollte sich im 18. Jahrhundert durchsetzen. Hermann Friedrich Teichmeyer (1723) unterscheidet einen weiten gerichtsmedizinischen Wundenbegriff von einem geläufigen engeren. Und tödlich sind für ihn alle Wunden, welche, wie er schreibt, die hydraulisch-pneumatisch-vitale Maschinerie unseres Körpers 
verletzen und deren Ökonomie so stören, daß niemand ihren Ruin verhindern kann ${ }^{20}$. Grundsätzlich dasselbe Verständnis findet man, mehr oder minder modifiziert, in den Werken von Johann Ernst Hebenstreit (1751), Wilhelm Gottfried Ploucquet (1777?), Joseph Jakob Plenk (1782) ${ }^{21}$ und Johann Daniel Metzger (1793). Tödlich sind für Metzger Verletzungen (Wunden, Quetschungen, Erschütterungen usw.), welche Lebensfunktionen (wie Respiration, Zirkulation, Chylifikation, gewisse Nervenfunktionen) «dergestalt stören, daß solche unmöglich wieder hergestellt werden können». ${ }^{22}$

Soviel zur wissenschaftlichen Entwicklung der gerichtsmedizinischen Wundbegutachtung. Ihr standespolitisches Äquivalent ist das allmähliche Übergehen der Wundbegutachtung aus den Händen der handwerklich orientierten Chirurgen in die Kompetenz medizinisch Gebildeter - seien dies nun chirurgisch gebildete Mediziner oder medizinisch gebildete Chirurgen. Doch dies sei hier platzeshalber nur angedeutet.

\section{Anmerkungen}

1 Zit. n. Celsus, Aulus Cornelius: Über die Arzneiwissenschaft in acht Büchern, Übers. v. E.Scheller, 2. Aufl., neu durchges. v. W. Frieboes, Braunschweig: Vieweg 1906, S.261 (Buch 5, Kap. 26,1).

2 Im früheren 17.Jahrhundert setzt Sebitz Tödlichkeit und Unheilbarkeit noch gleich, im späteren macht Bohn, der mit der prognostischen Tradition der Wundbegutachtung am Toten gebrochen hat, auf den Unterschied zwischen beidem ausdrücklich aufmerksam; Sebizius, Melchior: Prodromi examinis vulnerum singularum humani corporis partium, quatenus vel lethalia sunt et incurabilia: vel ratione eventus salutaria et sanabilia. Pars I: Vulnerum nomenclaturas, definitiones, differentias ... explicans. Resp. Dominicus Chabraeus, Argentorati: Welperus 1632, Titel:

Bohnius, Johannes: De renunciatione vulnerum, seu vulnerum lethalium examen, exponens horum formalitatem et causas, tam in genere, quam in specie ac per singulas corporis partes. Amstelodami: Vidua Sebastiani et Christianus Petzoldus 1710 (1. Ausg. Leipzig 1689). S. 18-19.

${ }^{3}$ Vgl. Sudhoff. Karl: Lehr- und Merkschemata für die Beurteilung der Schwere von Verletzungen ... In: Beiträge zur Geschichte der Chirurgie im Mittelalter. Graphische und textliche Untersuchungen in mittelalterlichen Handschriften. 1. Teil. Leipzig: Barth 1914 (Studien zur Geschichte der Medizin: 10), S.67-224. Tafel XIV:

Herrlinger. Robert: Geschichte der medizinischen Abbildung. 1: Von der Antike bis um 1600. Mïnchen: Moos 1967. S.37--38.

+ Kantorowiez. Hermann I.: Albertus Gandinus und das Strafrecht der Scholastik: 1.Bd.: Die Praxis. Berlin: Guttentag 1907. S.233. «in petine» (sollte wohl «in pectine» heißen) hier frei als «im Unterbauch» übersetzt. 
Für ähnliche Fälle, in denen die ärztlichen Experten die Wunden in derselben Weise nach Zahl und äußerer Topographie beschrieben und in tödliche und nichttödliche unterteilt haben vgl. Münster, Ladislao: La medicina legale a Bologna nel Quattrocento. Actes du $8^{e}$ Congrès International d'Histoire des Sciences, Firenze 3-9 Settembre 1956, S.687-711;

Bohne, Gotthold: Die gerichtliche Medizin im italienischen Statutarrecht des 13.-16. Jahrhunderts. (Ein Beitrag zur Geschichte der Medizin im Mittelalter.) Vjschr. gerichtl. Med. 61 (1921) 3. Folge, S. 81.

${ }^{5}$ Paré Ambroise: Le vingt-septième livre, traitant des rapports... In: Euvres complètes, t. 3, Nachdr. d. Ausg. Paris 1841, Genève: Slatkine Reprints 1970, S.651.

${ }^{6}$ Suevus, Bernhardus: Tractatus de inspectione vulnerum lethalium et sanabilium praecipuarum partium corporis humani, Marpurgi: Chemlinus 1629, S. 1-3 (I, Kap. 1).

7 Sebizius (Anm.2), praefatio.

${ }^{8}$ Celsus (Anm. 1), S. 263 (Buch 5, Kap. 26,6);

Paré (Anm. 5), S. 652-653.

${ }^{9}$ Codronchius, Baptista: Methodus testificandi. In: De vitiis vocis, libri duo, Francofurti: Wechelius 1597, S. 148-232.

${ }^{10}$ Fidelis, Fortunatus: De relationibus medicorum libri quatuor. Studio Pauli Ammanni, Lipsiae: Tarnovius 1674 (1. Ausg. Palermo 1602).

11 Codronchius (Anm. 9), S. 174.

12 Fidelis (Anm. 10), S. 526-529, 531-541 (Kap.3). 542-546 (Kap.4). Zum Beispiel beschreibt Fidelis die Zeichen der Herzverletzung weitgehend mit denselben Worten wie Celsus.

13 Bohnius (Anm.2), S. 4-5.

14 Hartmann, Fritz: Begründung des Themas ... [Ausführungen zur Einführung des 16. Symposiums der Gesellschaft für Wissenschaftsgeschichte «Prognose und Wissenschaft», Hannover 4.-6. Mai 1978] Ber. Wiss. Gesch. 2 (1979) 3-12, S. 4.

${ }^{15}$ Michler, Markwart: Einführung. In: Giovanni Battista Morgani, Sitz und Ursachen der Krankheiten, ausgew., übertr. u. eingel. v. Markwart Michler. Bern/Stuttgart: Huber 1967, S. 25-26.

16 Vgl. Codronchius (Anm.9), S.171;

Fidelis (Anm. 10), S.528.

17 Vgl. Sebitzius (Anm.5), Pars III: Problemata quaedam decidens. Resp. Joannes Guilielmus Zachmannus. Argentorati: Welperus 1633, § 80-81;

Welsch, Gottfried: Rationale vulnerum lethalium judicium. Lipsiae: Ritzschianus 1662 (1. Ausg. ebenda 1660), S. 7-16. Welsch betont bereits, daß die «Nobilitas» von Hirn, Herz und Leber auf deren «actio» beruhe.

18 Vgl. Welsch (Anm. 17), S.6-7.

19 Bohnius (Anm.2), S. 15-16.

${ }^{20}$ Teichmeyerus, Hermannus Fridericus: Institutiones medicinae legalis vel forensis. Jenae: Bielckius 1723, S. 188-191.

${ }^{21}$ Hebenstreit, Io. Ernestus: Anthropologia forensis. Ed. altera, Lipsiae: Lankisiani 1753, S. 342-588 (1. Ausg. Leipzig 1751);

Ploucquet, Wilhelm Gottfried: Anhandlung über die gewaltsame Todesarten. Tübingen: Berger o.J. (1777 nach Wilhelm Engelmann, Bibliotheca medica-chirurgica, Leipzig: Engelmann 1848), S.11-12;

Plenk, Joseph Jakob: Anfangsgründe der gerichtlichen Arztneywissenschaft und Wund- 
arztneykunst. Aus d. Lat. übers. v. F.August von Wasserberg, Wien: Gräffer 1782, S.28-29.

22 Metzger, Johann Danicl: Kurzgefaßtes System der gerichtlichen Arzneiwissensehaft. Königsberg-Leipzig: Hartung 1793, S. 48-50,67-70.

Vgl. auch Fodéré, François-Emmanuel: Les lois éclairées par les seiences physiques; ou traité de médecine-légale et d'hygiène publique, t.2, Paris: Croullebois/Deterville an 7 (1799), S.53: «En Médecine-légale, ... on appelle blessure toute lésion faite avec violence au corps humain, de laquelle puisse résulter une commotion, une solution de continuité, une contusion, une fracture, une brûlure, une déchirure, une torsion ou une luxation.»

\section{Summary}

When death from wounding was brought to court from the 16th to the 18th century, the medico-legal question would be: were the wounds under consideration deadly or not? how deadly were they? This implied the question of the offender's responsibility for the outcome of his deed.

At first, the experts used to answer from a prognostic point of view, later from an anatomical one, finally a physiological point of view prevailed.

Prof. Dr.med. Esther Fischer-Homberger

Medizinhistorisches Institut

Bühlstraße 26

C.H-3012 Bern 